\title{
Some Extensions of Fixed Point Results over Quasi-JS-Spaces
}

\author{
Maha Noorwali, ${ }^{1}$ Hamed H. Alsulami, ${ }^{1}$ and Erdal Karapınar ${ }^{2}$ \\ ${ }^{1}$ Nonlinear Analysis and Applied Mathematics Research Group (NAAM), Mathematics Department, Faculty of Science, \\ King Abdulaziz University, P.O. Box 42805, Jeddah 21551, Saudi Arabia \\ ${ }^{2}$ Department of Mathematics, Atilim University, 06836 Incek, Ankara, Turkey
}

Correspondence should be addressed to Maha Noorwali; mnorwali@kau.edu.sa

Received 7 November 2015; Accepted 6 April 2016

Academic Editor: Richard I. Avery

Copyright (C) 2016 Maha Noorwali et al. This is an open access article distributed under the Creative Commons Attribution License, which permits unrestricted use, distribution, and reproduction in any medium, provided the original work is properly cited.

We introduce the notion of quasi-JS-metric space. After defining the basic topological properties of quasi-JS-metric space, we investigate fixed point of certain mapping in the frame of complete quasi-JS-metric space. Our results unify and cover several existing fixed point theorems in distinct structures (such as standard quasi-metric spaces, quasi- $b$-metric spaces, dislocated quasimetric spaces, and quasi-modular spaces) in the literature.

\section{Introduction and Preliminaries}

Jleli and Samet [1] combined a number of existing fixed point results, by introducing a new distance (that includes, as particular cases, standard metric spaces, $b$-metric spaces, dislocated metric spaces, and modular spaces). In this paper, our aim is to refine the new distance by omitting a symmetry condition. Hence, our new approaches cover and combine several more interesting existing fixed point results (that includes, as particular cases, standard quasi-metric spaces, quasi- $b$-metric spaces, dislocated quasi-metric spaces, and quasi-modular spaces) including the results of Jleli and Samet [1].

For the sake of completeness, we collect some basic concepts and results from the literature. Let $\mathbb{N}_{0}$ denote the set $\mathbb{N} \cup\{0\}$ where $\mathbb{N}$ represent the set of all positive integers. Let $X$ be a nonempty set and let $\mathscr{D}: X \times X \rightarrow[0, \infty]$ be a given mapping. For every $x \in X$, define the sets

$$
\begin{aligned}
& C_{L}(\mathscr{D}, X, x)=\left\{\left\{x_{n}\right\} \subseteq X: \lim _{n \rightarrow \infty} \mathscr{D}\left(x, x_{n}\right)=0\right\}, \\
& C_{R}(\mathscr{D}, X, x)=\left\{\left\{x_{n}\right\} \subseteq X: \lim _{n \rightarrow \infty} \mathscr{D}\left(x_{n}, x\right)=0\right\} .
\end{aligned}
$$

Definition 1. We say that $\mathscr{D}: X \times X \rightarrow[0, \infty]$ is a quasi$J S$-metric space on a nonempty set $X$ if it fulfils the following conditions:

$\left(\mathrm{D}_{1}\right) \mathscr{D}(x, y)=\mathscr{D}(y, x)=0 \Rightarrow x=y$, for every $x, y \in X$.
$\left(\mathrm{D}_{2}\right)$ There exists $C>0$ such that

$$
\begin{aligned}
& \text { if } x, y \in X,\left\{x_{n}\right\} \in C_{L}(\mathscr{D}, X, x), \\
& \text { then } \mathscr{D}(x, y) \leq C \limsup _{n \rightarrow \infty} \mathscr{D}\left(x_{n}, y\right), \\
& \text { if } x, y \in X,\left\{x_{n}\right\} \in C_{R}(\mathscr{D}, X, x), \\
& \text { then } \mathscr{D}(y, x) \leq C \limsup _{n \rightarrow \infty} \mathscr{D}\left(y, x_{n}\right) .
\end{aligned}
$$

In this case, the pair $(X, \mathscr{D})$ is called a quasi- $J S$-metric space.

Remark 2. If, in addition to the conditions in Definition 1, the equality,

$$
\left(\mathrm{D}_{3}\right) \mathscr{D}(x, y)=\mathscr{D}(y, x),
$$

is satisfied for each $x, y \in X$, then, $(X, \mathscr{D})$ is called $J S$-metric space [1].

In what follows we shall define some basic topological notions for quasi- $J S$-metric space.

Definition 3. Let $(X, \mathscr{D})$ be a quasi-JS-metric space. Let $\left\{x_{n}\right\}$ be a sequence in $X$ and $x \in X$. Then,

(i) $\left\{x_{n}\right\}$ is said to be left $\mathscr{D}$-convergent to $x$ if $\left\{x_{n}\right\} \in$ $C_{L}(\mathscr{D}, X, x)$; in this case $x$ is said to be a left $\mathscr{D}$-limit of $\left\{x_{n}\right\}$. 
(ii) $\left\{x_{n}\right\}$ is said to be right $\mathscr{D}$-convergent to $x$ if $\left\{x_{n}\right\} \in$ $C_{R}(\mathscr{D}, X, x)$; in this case $x$ is said to be a right $\mathscr{D}$-limit of $\left\{x_{n}\right\}$.

(iii) $\left\{x_{n}\right\}$ is said to be $\mathscr{D}$-convergent to $x$ if $x_{n}$ is both left and right $\mathscr{D}$-convergent to $x$; in this case $x$ is said to be a $\mathscr{D}$-limit of $\left\{x_{n}\right\}$ (see [1]).

Proposition 4. The D-limit of any sequence in a quasi-JSmetric space is unique.

Proof. Let $(X, \mathscr{D})$ be a quasi-JS-metric space. Let $\left\{x_{n}\right\}$ be a sequence in $X$. Assume that $x, y$ are both $\mathscr{D}$-limits of $\left\{x_{n}\right\}$. On account of $\left(\mathrm{D}_{2}\right)$ and regarding the definition of $\mathscr{D}$ convergence, we find that

$$
\begin{aligned}
& \mathscr{D}(y, x) \leq C \limsup _{n \rightarrow \infty} \mathscr{D}\left(y, x_{n}\right)=0, \\
& \quad \text { since }\left\{x_{n}\right\} \in C_{R}(\mathscr{D}, X, x),\left\{x_{n}\right\} \in C_{L}(\mathscr{D}, X, y)
\end{aligned}
$$

and also

$$
\begin{aligned}
& \mathscr{D}(x, y) \leq C \limsup _{n \rightarrow \infty} \mathscr{D}\left(x, x_{n}\right)=0, \\
& \quad \text { since }\left\{x_{n}\right\} \in C_{R}(\mathscr{D}, X, y),\left\{x_{n}\right\} \in C_{L}(\mathscr{D}, X, x) .
\end{aligned}
$$

Thus, we have $\mathscr{D}(x, y)=0=\mathscr{D}(y, x)$. By $\left(\mathrm{D}_{1}\right)$, we find $x=$ $y$.

Definition 5. Let $(X, \mathscr{D})$ be a quasi-JS-metric space. Let $\left\{x_{n}\right\}$ be a sequence in $X$.

(i) $\left\{x_{n}\right\}$ is said to be left $\mathscr{D}$-Cauchy sequence if

$$
\lim _{m, n \rightarrow \infty} \mathscr{D}\left(x_{m+n}, x_{n}\right)=0 .
$$

(ii) $\left\{x_{n}\right\}$ is said to be right $\mathscr{D}$-Cauchy sequence if

$$
\lim _{m, n \rightarrow \infty} \mathscr{D}\left(x_{n}, x_{m+n}\right)=0 .
$$

(iii) $\left\{x_{n}\right\}$ is said to be $\mathscr{D}$-Cauchy sequence if it is both left and right $\mathscr{D}$-Cauchy sequence (see [1]).

Definition 6. Let $(X, \mathscr{D})$ be a quasi-JS-metric space.

(i) $X$ is said to be left $\mathscr{D}$-complete if every left $\mathscr{D}$-Cauchy sequence in $X$ is left $\mathscr{D}$-convergent to some element in $X$.

(ii) $X$ is said to be right $\mathscr{D}$-complete if every right $\mathscr{D}$ Cauchy sequence in $X$ is right $\mathscr{D}$-convergent to some element in $X$.

(iii) $X$ is said to be $\mathscr{D}$-complete if and only if it is left and right $\mathscr{D}$-complete, so that every $\mathscr{D}$-Cauchy sequence in $X$ is $\mathscr{D}$-convergent to some element in $X$ (see [1]).

Example 7. Let $X=\mathbb{R} \cup\{\infty\}$ and define $\mathscr{D}: X \times X \rightarrow[0, \infty]$ by

$$
\mathscr{D}(x, y)= \begin{cases}\infty & \text { if } x \text { or } y=\infty \\ |x-y| & \text { otherwise. }\end{cases}
$$

Then, clearly, $\mathscr{D}$ satisfies $\left(\mathrm{D}_{1}\right)$. Let $x \in X$; we have two cases:

Case 1: if $x=\infty$, then $C_{L}(\mathscr{D}, X, x)=C_{R}(\mathscr{D}, X, x)=$ $\emptyset$.

Case 2: if $x<\infty$, let $\left\{z_{n}\right\} \in C_{L}(\mathscr{D}, X, x)$; then $\lim _{n \rightarrow \infty} \mathscr{D}\left(x, z_{n}\right)=0$ and so $z_{n}<\infty$ except possibly for finite number of terms. Let $m$ be the smallest natural number such that $z_{n}<\infty$ for all $n \geq m$. Now, let $y \in X$; if $y=\infty$, then

$$
\mathscr{D}(x, y)=\infty=\limsup _{n \rightarrow \infty} \mathscr{D}\left(z_{n}, y\right) .
$$

On the other hand, if $y<\infty$, then we get

$$
\mathscr{D}(x, y)=|x-y| \leq\left|x-z_{n}\right|+\left|z_{n}-y\right| \quad \forall n \geq m .
$$

Thus, we find that

$$
\mathscr{D}(x, y)=|x-y| \leq \limsup _{n \rightarrow \infty} \mathscr{D}\left(z_{n}, y\right) .
$$

Similarly, if $\left\{x_{n}\right\} \in C_{R}(\mathscr{D}, X, x)$, then for any $y \in X$ we have

$$
\mathscr{D}(y, x) \leq \limsup _{n \rightarrow \infty} \mathscr{D}\left(y, x_{n}\right) .
$$

Consequently, $\mathscr{D}$ satisfies condition $\left(\mathrm{D}_{2}\right)$ with $C=1$. Therefore, $(X, \mathscr{D})$ is a quasi-JS-metric space. Now, let $\left\{x_{n}\right\} \subseteq$ $X$ be $\mathscr{D}$-Cauchy sequence. Then there exists a smallest $m \in \mathbb{N}$ such that $x_{n}<\infty$ for all $n \geq m$. As the restriction of $\mathscr{D}$ to $\mathbb{R}$ is just the usual metric on $\mathbb{R},\left\{x_{n}\right\}$ is $\mathscr{D}$-convergent to some $x \in X$. Thus, $(X, \mathscr{D})$ is a complete quasi-JS-metric space.

Definition 8. Let $X$ be a nonempty set. A mapping $q: X \times$ $X \rightarrow[0, \infty)$ is called quasi-metric on $X$, if the following conditions are fulfilled:

$\left(\mathrm{Q}_{1}\right)$ for every $x, y \in X$, we have $q(x, y)=0=q(y, x) \Leftrightarrow$ $x=y$;

$\left(\mathrm{Q}_{2}\right)$ for every $x, y, z \in X$, we have $q(x, y) \leq q(x, z)+$ $q(z, y)$.

Here, the pair $(X, q)$ is called quasi-metric space.

Proposition 9. Any quasi-metric space is a quasi-JS-metric space with $C=1$.

Proof is straightforward, so we omit it.

Example 10. Let $X$ be a set and let $f: X \rightarrow[0, \infty)$ be an arbitrary one to one function. Set

$$
q(x, y)=\max \{f(y)-f(x), 0\} \quad \forall x, y \in X .
$$

Then $q$ is a quasi-metric space [2].

In 2012, Shah and Hussain [3] introduced the concept of quasi- $b$-metric spaces and verified some fixed point theorems in quasi- $b$-metric spaces. 
Definition 11. Let $X$ be a nonempty set, let $s \geq 1$ be a given real number, and let $d: X \times X \rightarrow[0, \infty)$ be a mapping satisfying the following conditions:

$\left(\mathrm{QBM}_{1}\right)$ for every $x, y \in X$, we have $d(x, y)=0 \Leftrightarrow$ $x=y$;

$\left(\mathrm{QBM}_{2}\right)$ for every $x, y, z \in X$, we have $d(x, y) \leq$ $s[d(x, z)+d(z, y)]$.

Then, $d$ is said to be a quasi- $b$-metric space, and $(X, d)$ is called quasi- $b$-metric space.

The following proposition followed immediately from the previous definition.

Proposition 12. Any quasi-b-metric space is a quasi-JS-metric space with $C=s$.

Proof. Let $(X, d)$ be a quasi-b-metric space. Since the first condition is straightforward, it is sufficient to show that $d$ fulfils the property $\left(\mathrm{D}_{2}\right)$ of Definition 1 . Let $x \in X$ and $\left\{x_{n}\right\} \in C_{L}(d, X, x)$. For every $y \in X$, by the property $\left(\mathrm{QBM}_{2}\right)$, we have

$$
d(x, y) \leq s d\left(x, x_{n}\right)+s d\left(x_{n}, y\right),
$$

for each $n$. Thus we have

$$
d(x, y) \leq s \limsup _{n \rightarrow \infty} d\left(x_{n}, y\right) .
$$

Analogously, for the case $\left\{x_{n}\right\} \in C_{R}(d, X, x)$, we derive that

$$
d(y, x) \leq s \limsup _{n \rightarrow \infty} d\left(y, x_{n}\right) .
$$

In 2005, Zeyada et al. [4] introduced the concept of complete dislocated quasi-metric space and obtain some fixed point results on it.

Definition 13. Let $X$ be a nonempty set and $d: X \times X \rightarrow$ $[0, \infty), d$ is said to be dislocated quasi-metric (or quasimetric-like) if it satisfies the following conditions for every $x, y, z \in X$ :

$$
\begin{aligned}
& \left(\mathrm{QML}_{1}\right) d(x, y)=0=d(y, x) \Rightarrow x=y ; \\
& \left(\mathrm{QML}_{2}\right) d(x, y) \leq d(x, z)+d(z, y) .
\end{aligned}
$$

In this case $(X, d)$ is called dislocated quasi-metric space (or quasi-metric-like space). If in addition $d$ satisfies

$$
\left(\mathrm{QML}_{3}\right) d(x, y)=d(y, x) \text { for every } x, y \in X,
$$

then it is called dislocated metric.

Proposition 14. Any dislocated quasi-metric space is a quasiJS-metric space.

Example 15. Let $X=[0,1]$ and define $q: X \times X \rightarrow[0, \infty)$ by

$$
q(x, y)=|x-y|+x \quad \text { for each } x, y \in X .
$$

Then $(X, q)$ is a dislocated quasi-metric.
In 1988, Kozlowski introduced the notion of modular spaces [5]; before we generalize this notion to the quasi form we need the following definitions.

Definition 16. Let $X$ be a linear space over $\mathbb{R}$. A function $\rho: X \rightarrow[0, \infty]$ is said to be quasi-modular if the following conditions hold:

$$
\left(q \rho_{1}\right) \rho(x)=0 \Leftrightarrow x=0 ;
$$

$\left(q \rho_{2}\right)$ for every $x, y \in X$, we have

$$
\rho(\alpha x+\beta y) \leq \rho(x)+\rho(y)
$$

whenever $\alpha, \beta \geq 0$ and $\alpha+\beta=1$. If in addition $\rho$ satisfies

$$
\rho(-x)=\rho(x) \quad \text { for every } x \in X,
$$

then $\rho$ is called modular on $X$.

Definition 17. Let $X$ be a linear space and let $\rho: X \rightarrow[0, \infty]$ be a quasi-modular space on $X$. The set

$$
X_{\rho}=\left\{x \in X: \lim _{\lambda \rightarrow 0} \rho(\lambda x)=0\right\}
$$

is called a quasi-modular space.

The convergence in quasi-modular spaces is defined as follows.

Definition 18. Let $X_{\rho}$ be a quasi-modular space, let $\left\{x_{n}\right\}$ be a sequence in $X_{\rho}$, and $x \in X_{\rho}$.

(i) $\left\{x_{n}\right\}$ is said to be left $\rho$-convergent to $x \in X$ if $\lim _{n \rightarrow \infty} \rho\left(x-x_{n}\right)=0$.

(ii) $\left\{x_{n}\right\}$ is said to be right $\rho$-convergent to $x \in X$ if $\lim _{n \rightarrow \infty} \rho\left(x_{n}-x\right)=0$.

(iii) $\left\{x_{n}\right\}$ is said to be $\rho$-convergent to $x \in X$ if it is both left and right $\rho$-convergent to $x$.

Definition 19. Let $X_{\rho}$ be a quasi-modular space and let $\left\{x_{n}\right\}$ be a sequence in $X_{\rho}$.

(i) $\left\{x_{n}\right\}$ is said to be left $\rho$-Cauchy if $\lim _{m, n \rightarrow \infty} \rho\left(x_{m+n}-\right.$ $\left.x_{n}\right)=0$.

(ii) $\left\{x_{n}\right\}$ is said to be right $\rho$-Cauchy if $\lim _{m, n \rightarrow \infty} \rho\left(x_{n}-\right.$ $\left.x_{m+n}\right)=0$.

(iii) $\left\{x_{n}\right\}$ is said to be $\rho$-Cauchy if it is both left and right $\rho$-Cauchy.

Definition 20. (i) A quasi-modular space $X_{\rho}$ is said to be left (right) $\rho$-complete if every left (right) $\rho$-Cauchy sequence converges to some $x \in X_{\rho}$.

(ii) A quasi-modular space $X_{\rho}$ is said to be $\rho$-complete if and only if it is both left and right $\rho$-complete. 
Definition 21. Let $X_{\rho}$ be a quasi-modular space.

(i) $\rho$ is said to have left Fatou property if for every $y \in X_{\rho}$

$$
\rho(y-x) \leq \liminf _{n \rightarrow \infty} \rho\left(y-x_{n}\right)
$$

whenever $\left\{x_{n}\right\} \subseteq X_{\rho}$ is left $\rho$-convergent to $x \in X_{\rho}$.

(ii) $\rho$ is said to have right Fatou property if for every $y \in$ $X_{\rho}$

$$
\rho(x-y) \leq \liminf _{n \rightarrow \infty} \rho\left(x_{n}-y\right)
$$

whenever $\left\{x_{n}\right\} \subseteq X_{\rho}$ is right $\rho$-convergent to $x \in X_{\rho}$.

(iii) $\rho$ is said to have Fatou property if it has left and right Fatou property.

Let $X_{\rho}$ be a quasi-modular space. Define the mapping $\mathscr{D}_{\rho}: X_{\rho} \times X_{\rho} \rightarrow[0, \infty]$ by

$$
\mathscr{D}_{\rho}(x, y)=\rho(x-y) \quad \forall x, y \in X_{\rho} .
$$

We have the following example of a quasi-JS-space.

Proposition 22. Let $X_{\rho}$ be a quasi-modular space such that $\rho$ has Fatou property. Then $\mathscr{D}_{\rho}$ is quasi-JS-metric on $X_{\rho}$ with $C=1$.

Proof. Clearly, $\mathscr{D}_{\rho}$ satisfies $\left(\mathrm{D}_{1}\right)$. Let us prove that $\mathscr{D}_{\rho}$ satisfies $\left(\mathrm{D}_{2}\right)$. Let $x \in X_{\rho}$ and let $\left\{x_{n}\right\} \in C_{L}\left(\mathscr{D}_{\rho}, X_{\rho}, x\right)$. As $\rho$ has Fatou property for any $y \in X_{\rho}$ we have

$$
\begin{aligned}
\mathscr{D}_{\rho}(x, y) & =\rho(x-y) \leq \liminf _{n \rightarrow \infty} \rho\left(x_{n}-y\right) \\
& \leq \limsup _{n \rightarrow \infty} \rho\left(x_{n}-y\right) \\
& =\limsup _{n \rightarrow \infty} \mathscr{D}_{\rho}\left(x_{n}, y\right) .
\end{aligned}
$$

Similarly, if $\left\{x_{n}\right\} \in C_{R}\left(\mathscr{D}_{\rho}, X_{\rho}, x\right)$, then for every $y \in X_{\rho}$ we have

$$
\begin{aligned}
\mathscr{D}_{\rho}(y, x) & =\rho(y-x) \leq \liminf _{n \rightarrow \infty} \rho\left(y-x_{n}\right) \\
& \leq \limsup _{n \rightarrow \infty} \rho\left(y-x_{n}\right) \\
& =\limsup _{n \rightarrow \infty} \mathscr{D}_{\rho}\left(y, x_{n}\right) .
\end{aligned}
$$

Thus, $\mathscr{D}_{\rho}$ is a quasi-JS-metric space on $X_{\rho}$.

Consequently, we have the following result.

Proposition 23. Let $X_{\rho}$ be a quasi-modular space where $\rho$ has Fatou property. Then

(i) a sequence $\left\{x_{n}\right\} \subseteq X_{\rho}$ is left $\rho$-convergent (right $\rho$ convergent or $\rho$-convergent) to some $x \in X_{\rho}$ if and only if it is left $\mathscr{D}_{\rho}$-convergent (right $\mathscr{D}_{\rho}$-convergent or $\mathscr{D}_{\rho^{-}}$ convergent) to $x$; (ii) a sequence $\left\{x_{n}\right\} \subseteq X_{\rho}$ is left $\rho$-Cauchy (right $\rho$-Cauchy or $\rho$-Cauchy) if and only if it is left $\mathscr{D}_{\rho}$-Cauchy (right $\mathscr{D}_{\rho}$-Cauchy or $\mathscr{D}_{\rho}$-Cauchy);

(iii) $X_{\rho}$ is left $\rho$-complete (right $\rho$-complete or $\rho$-complete) if and only if it is left $\mathscr{D}_{\rho}$-complete (right $\mathscr{D}_{\rho}$-complete or $\mathscr{D}_{\rho}$-complete).

\section{The Banach Contraction Principle in a Quasi-JS-Metric Space}

The Banach contraction principle was extended to a $J S$ metric space by Jleli and Samet in [1]. We shall extend this principle to a quasi-JS-metric space.

Definition 24. Let $(X, \mathscr{D})$ be a quasi-JS-metric space and let $f: X \rightarrow X$ be a function. We say that $f$ is $k$-contraction if

$$
\mathscr{D}(f(x), f(y)) \leq k \mathscr{D}(x, y),
$$

for every $x, y \in X$, where $k \in(0,1)$.

Proposition 25. Let $(X, \mathscr{D})$ be a quasi-JS-metric space. Suppose that the function $f: X \rightarrow X$ is $k$-contraction for some $k \in(0,1)$. Then any fixed point $\omega \in X$ of $f$ with $\mathscr{D}(\omega, \omega)<\infty$ satisfies $\mathscr{D}(\omega, \omega)=0$.

Proof. Let $\omega \in X$ be a fixed point of $f$ with $\mathscr{D}(\omega, \omega)<\infty$. Then, as $f$ is $k$-contraction, we have

$$
\mathscr{D}(\omega, \omega)=\mathscr{D}(f(\omega), f(\omega)) \leq k \mathscr{D}(\omega, \omega),
$$

which is possible only if $\mathscr{D}(\omega, \omega)=0$.

For each $x \in X$ let us define

$$
\delta(\mathscr{D}, f, x)=\sup \left\{\mathscr{D}\left(f^{i}(x), f^{j}(x)\right): i, j \in \mathbb{N}_{0}\right\},
$$

where $f^{0}(x)=x$.

The following theorem is an extension of Banach contraction principle in the context of a quasi-JS-metric space.

Theorem 26. Let $(X, \mathscr{D})$ be a $\mathscr{D}$-complete quasi-JS-metric space and let $f: X \rightarrow X$ be a $k$-contraction mapping for some $k \in(0,1)$. Suppose that there exists $x_{0} \in X$ such that $\delta\left(\mathscr{D}, f, x_{0}\right)<\infty$. Then, $f$ has a fixed point $\omega \in X$ and $\left\{f^{n}\left(x_{0}\right)\right\}$ is $\mathscr{D}$-convergent to $\omega$. Moreover, if $\omega^{\prime}$ is another fixed point of $f$ such that $\mathscr{D}\left(\omega, \omega^{\prime}\right)<\infty$ and $\mathscr{D}\left(\omega^{\prime}, \omega\right)<\infty$, then $\omega=\omega^{\prime}$.

Proof. We shall prove that $\left\{f^{n}\left(x_{0}\right)\right\}$ is a $\mathscr{D}$-Cauchy sequence. Let $n \in \mathbb{N}$, as $f$ is $k$-contraction, for each $i, j \in \mathbb{N}$ we have

$$
\begin{aligned}
& \mathscr{D}\left(f^{n+i}\left(x_{0}\right), f^{n+j}\left(x_{0}\right)\right) \\
& \quad \leq k \mathscr{D}\left(f^{n-1+i}\left(x_{0}\right), f^{n-1+j}\left(x_{0}\right)\right),
\end{aligned}
$$

which implies that

$$
\delta\left(\mathscr{D}, f, f^{n}\left(x_{0}\right)\right) \leq k \delta\left(\mathscr{D}, f, f^{n-1}\left(x_{0}\right)\right) .
$$

So, we obtain that

$$
\delta\left(\mathscr{D}, f, f^{n}\left(x_{0}\right)\right) \leq k^{n} \delta\left(\mathscr{D}, f, x_{0}\right) .
$$


Taking (30) into account and regarding the definition of $\delta$, for every $m, n \in \mathbb{N}_{0}$, we have

$$
\begin{aligned}
\mathscr{D} & \left(f^{n}\left(x_{0}\right), f^{n+m}\left(x_{0}\right)\right) \leq \delta\left(\mathscr{D}, f, f^{n}\left(x_{0}\right)\right) \\
& \leq k^{n} \delta\left(\mathscr{D}, f, x_{0}\right) .
\end{aligned}
$$

Using the fact that $\delta\left(\mathscr{D}, f, x_{0}\right)<\infty$ and $k \in(0,1)$ we have

$$
\lim _{m, n \rightarrow \infty} \mathscr{D}\left(f^{n}\left(x_{0}\right), f^{n+m}\left(x_{0}\right)\right)=0,
$$

which implies that $\left\{f^{n}\left(x_{0}\right)\right\}$ is right $\mathscr{D}$-Cauchy.

Analogously, we have

$$
\begin{aligned}
& \mathscr{D}\left(f^{n+m}\left(x_{0}\right), f^{n}\left(x_{0}\right)\right) \leq \delta\left(\mathscr{D}, f, f^{n}\left(x_{0}\right)\right) \\
& \quad \leq k^{n} \delta\left(\mathscr{D}, f, x_{0}\right),
\end{aligned}
$$

which implies

$$
\lim _{m, n \rightarrow \infty} \mathscr{D}\left(f^{n+m}\left(x_{0}\right), f^{n}\left(x_{0}\right)\right)=0 .
$$

Thus, $\left\{f^{n}\left(x_{0}\right)\right\}$ is left $\mathscr{D}$-Cauchy and, hence, it is $\mathscr{D}$-Cauchy sequence in $(X, \mathscr{D})$. By completeness of $(X, \mathscr{D})$ there exists $\omega \in X$ such that $\lim _{n \rightarrow \infty} f^{n}\left(x_{0}\right)=\omega$. Since $f$ is $k$ contraction, we have

$$
\begin{aligned}
& \mathscr{D}\left(f^{n+1}\left(x_{0}\right), f(\omega)\right) \leq k \mathscr{D}\left(f^{n}\left(x_{0}\right), \omega\right), \\
& \mathscr{D}\left(f(\omega), f^{n+1}\left(x_{0}\right)\right) \leq k \mathscr{D}\left(\omega, f^{n}\left(x_{0}\right)\right) .
\end{aligned}
$$

Hence $\lim _{n \rightarrow \infty} \mathscr{D}\left(f^{n+1}\left(x_{0}\right), f(\omega)\right)=0=\lim _{n \rightarrow \infty} \mathscr{D}(f(\omega)$, $\left.f^{n+1}\left(x_{0}\right)\right)=0$. So $f(\omega)$ is another $\mathscr{D}$-limit for the sequence $\left\{f^{n}\left(x_{0}\right)\right\}$. By the uniqueness of the limit in a quasi-JS-metric space (Proposition 4) we have $\omega=f(\omega)$. Now, if $\omega^{\prime}$ is another fixed point of $f$ with $\mathscr{D}\left(\omega, \omega^{\prime}\right)<\infty$, then, as $f$ is $k$-contraction, we have

$$
\mathscr{D}\left(\omega, \omega^{\prime}\right)=\mathscr{D}\left(f(\omega), f(\omega)^{\prime}\right) \leq k \mathscr{D}\left(\omega, \omega^{\prime}\right),
$$

which implies that $\mathscr{D}\left(\omega, \omega^{\prime}\right)=0$. Similarly, using the fact that $\mathscr{D}\left(\omega^{\prime}, \omega\right)<\infty$, we can prove that $\mathscr{D}\left(\omega^{\prime}, \omega\right)=0$. Therefore, $\omega=\omega^{\prime}$.

Since any quasi-metric space and any quasi- $b$-metric space is a quasi-JS-metric space, we derive the following results.

Corollary 27. Let $(X, q)$ be a complete quasi-metric space and let $f: X \rightarrow X$ be $k$-contraction mapping for some $k \in(0,1)$. Suppose that there exists $x_{0} \in X$ such that

$$
\sup \left\{q\left(f^{i}\left(x_{0}\right), f^{j}\left(x_{0}\right)\right): i, j \in \mathbb{N}_{0}\right\}<\infty .
$$

Then $f$ has a unique fixed point $\omega \in X$. Moreover, the sequence $\left\{f^{n}\left(x_{0}\right)\right\}$ converges to $\omega$.

Corollary 28. Let $(X, d)$ be a complete quasi-b-metric space and let $f: X \rightarrow X$ be $k$-contraction mapping for some $k \epsilon$ $(0,1)$. Suppose that there exists $x_{0} \in X$ such that

$$
\sup \left\{d\left(f^{i}\left(x_{0}\right), f^{j}\left(x_{0}\right)\right): i, j \in \mathbb{N}_{0}\right\}<\infty .
$$

Then $f$ has a unique fixed point $\omega \in X$. Moreover, the sequence $\left\{f^{n}\left(x_{0}\right)\right\}$ converges to $\omega$.
We can obtain a similar result in the context of complete dislocated quasi-metric spaces.

\section{3. Ćirić Type Contraction in a Quasi-JS-Metric Space}

In this section, we consider the existence and uniqueness of fixed point for Ćirić type contraction in the setting of quasi$J S$-metric space.

Definition 29. Let $f: X \rightarrow X$ be a function and $k \in(0,1)$. We say that $f$ is a generalized $k$-quasi-contraction mapping if it satisfies the following condition:

$$
\begin{aligned}
& (Q): \mathscr{D}(f(x), f(y)) \leq k \max \{\mathscr{D}(x, y), \\
& \mathscr{D}(x, f(x)), \mathscr{D}(y, f(y)), \mathscr{D}(x, f(y)), \\
& \mathscr{D}(y, f(x))\},
\end{aligned}
$$

for every $x, y \in X$.

Proposition 30. Suppose that $f$ is a generalized $k$-quasicontraction mapping for some $k \in(0,1)$. If $f$ has a fixed point $\omega \in X$ with $\mathscr{D}(\omega, \omega)<\infty$, then $\mathscr{D}(\omega, \omega)=0$.

Theorem 31. Let $(X, \mathscr{D})$ be a $\mathscr{D}$-complete quasi-JS-metric space with constant $C$ and let $f: X \rightarrow X$ be a generalized $k$-quasi-contraction mapping for some $k \in(0,1)$. Suppose that there exists $x_{0} \in X$ such that $\delta\left(\mathscr{D}, f, x_{0}\right)<\infty$. Then $\left\{f^{n}\left(x_{0}\right)\right\}$ converges to some $\omega \in X$. If $\mathscr{D}\left(x_{0}, f(\omega)\right)<\infty$, $\mathscr{D}(\omega, f(\omega))<\infty$, and $C<1$, then $\omega$ is a fixed point of $f$. Moreover, if $\omega^{\prime}$ is another fixed point of $f$ with $\mathscr{D}\left(\omega, \omega^{\prime}\right)<\infty$, $\mathscr{D}\left(\omega^{\prime}, \omega\right)<\infty$, and $\mathscr{D}\left(\omega^{\prime}, \omega^{\prime}\right)<\infty$, then $\omega=\omega^{\prime}$.

Proof. Let $n \in \mathbb{N}$, since $f$ is generalized $k$-quasi-contraction mapping, for all $i, j \in \mathbb{N}_{0}$; we have

$$
\begin{aligned}
& \mathscr{D}\left(f^{n+i}\left(x_{0}\right), f,^{n+j}\left(x_{0}\right)\right) \leq k \\
& \cdot \max \left\{\mathscr{D}\left(f^{n-1+i}\left(x_{0}\right), f^{n-1+j}\left(x_{0}\right)\right),\right. \\
& \mathscr{D}\left(f^{n-1+i}\left(x_{0}\right), f^{n+i}\left(x_{0}\right)\right), \\
& \mathscr{D}\left(f^{n-1+i}\left(x_{0}\right), f^{n+j}\left(x_{0}\right)\right), \\
& \mathscr{D}\left(f^{n-1+j}\left(x_{0}\right), f^{n+j}\left(x_{0}\right)\right), \\
& \left.\mathscr{D}\left(f^{n-1+j}\left(x_{0}\right), f^{n+i}\left(x_{0}\right)\right)\right\} .
\end{aligned}
$$

As

$$
\begin{aligned}
& \delta\left(\mathscr{D}, f, f^{n-1}\left(x_{0}\right)\right) \\
& \quad=\sup \left\{\mathscr{D}\left(f^{n-1+i}(x), f^{n-1+j}(x)\right): i, j \in \mathbb{N}_{0}\right\},
\end{aligned}
$$


we have

$$
\begin{aligned}
\max & \left\{\mathscr{D}\left(f^{n-1+i}\left(x_{0}\right), f^{n-1+j}\left(x_{0}\right)\right),\right. \\
& \mathscr{D}\left(f^{n-1+i}\left(x_{0}\right), f^{n+i}\left(x_{0}\right)\right), \\
& \mathscr{D}\left(f^{n-1+i}\left(x_{0}\right), f^{n+j}\left(x_{0}\right)\right), \\
& \mathscr{D}\left(f^{n-1+j}\left(x_{0}\right), f^{n+j}\left(x_{0}\right)\right), \\
& \left.\mathscr{D}\left(f^{n-1+j}\left(x_{0}\right), f^{n+i}\left(x_{0}\right)\right)\right\} \leq \delta\left(\mathscr{D}, f, f^{n-1}\left(x_{0}\right)\right) .
\end{aligned}
$$

Therefore, (40) yields $\delta\left(\mathscr{D}, f, f^{n}\left(x_{0}\right)\right) \leq k \delta\left(\mathscr{D}, f, f^{n-1}\left(x_{0}\right)\right)$. Hence for any $n \in \mathbb{N}_{0}$ we have

$$
\delta\left(\mathscr{D}, f, f^{n}\left(x_{0}\right)\right) \leq k^{n} \delta\left(\mathscr{D}, f, x_{0}\right) .
$$

Then for every $m, n \in \mathbb{N}_{0}$ we find

$$
\begin{aligned}
& \mathscr{D}\left(f^{n}\left(x_{0}\right), f^{n+m}\left(x_{0}\right)\right) \leq \delta\left(\mathscr{D}, f, f^{n}\left(x_{0}\right)\right) \\
& \quad \leq k^{n} \delta\left(\mathscr{D}, f, x_{0}\right), \\
& \mathscr{D}\left(f^{n+m}\left(x_{0}\right), f^{n}\left(x_{0}\right)\right) \leq \delta\left(\mathscr{D}, f, f^{n}\left(x_{0}\right)\right) \\
& \quad \leq k^{n} \delta\left(\mathscr{D}, f, x_{0}\right) .
\end{aligned}
$$

Since $\delta\left(\mathscr{D}, f, x_{0}\right)<\infty$ and $k<1$, we derive

$$
\begin{aligned}
\lim _{m, n \rightarrow \infty} \mathscr{D}\left(f^{n}\left(x_{0}\right), f^{n+m}\left(x_{0}\right)\right) \\
\quad=\lim _{m, n \rightarrow \infty} \mathscr{D}\left(f^{n+m}\left(x_{0}\right), f^{n}\left(x_{0}\right)\right)=0 .
\end{aligned}
$$

This implies that $\left\{f^{n}\left(x_{0}\right)\right\}$ is both left and right $\mathscr{D}$-Cauchy sequence and hence $\mathscr{D}$-Cauchy sequence. By completeness of $X$ there exists some $\omega \in X$ such that $\left\{f^{n}\left(x_{0}\right)\right\}$ converges to $\omega$; that is, $\lim _{n \rightarrow \infty} \mathscr{D}\left(f^{n}\left(x_{0}\right), \omega\right)=\lim _{n \rightarrow \infty} \mathscr{D}\left(\omega, f^{n}\left(x_{0}\right)\right)=0$.

Note that as in (44) for any $m, n \in \mathbb{N}_{0}$ we have

$$
\begin{aligned}
& \mathscr{D}\left(f^{n}\left(x_{0}\right), f^{m}\left(x_{0}\right)\right) \leq \delta\left(\mathscr{D}, f, f^{n}\left(x_{0}\right)\right) \\
& \quad \leq k^{n} \delta\left(\mathscr{D}, f, x_{0}\right), \\
& \mathscr{D}\left(f^{m}\left(x_{0}\right), f^{n}\left(x_{0}\right)\right) \leq \delta\left(\mathscr{D}, f, f^{n}\left(x_{0}\right)\right) \\
& \quad \leq k^{n} \delta\left(\mathscr{D}, f, x_{0}\right) .
\end{aligned}
$$

Now, assume that $\mathscr{D}(\omega, f(\omega))<\infty$ and $\mathscr{D}\left(x_{0}, f(\omega)\right)<$ $\infty$. Then, by using $(46)$ and condition $\left(\mathrm{D}_{2}\right)$ there exists $C>0$ such that

$$
\begin{aligned}
\mathscr{D}\left(\omega, f^{n}\left(x_{0}\right)\right) & \leq C \limsup _{m \rightarrow \infty} \mathscr{D}\left(f^{m}\left(x_{0}\right), f^{n}\left(x_{0}\right)\right) \\
& \leq C k^{n} \delta\left(\mathscr{D}, f, x_{0}\right) \\
\mathscr{D}\left(f^{n}\left(x_{0}\right), \omega\right) & \leq C \limsup _{m \rightarrow \infty} \mathscr{D}\left(f^{n}\left(x_{0}\right), f^{m}\left(x_{0}\right)\right) \\
& \leq C k^{n} \delta\left(\mathscr{D}, f, x_{0}\right)
\end{aligned}
$$

for every $n \in \mathbb{N}_{0}$.
On the other hand, as $f$ is generalized $k$-quasi-contraction mapping we have

$$
\begin{gathered}
\mathscr{D}\left(f\left(x_{0}\right), f(\omega)\right) \leq k \max \left\{\mathscr{D}\left(x_{0}, \omega\right), \mathscr{D}\left(x_{0}, f\left(x_{0}\right)\right),\right. \\
\left.\mathscr{D}(\omega, f(\omega)), \mathscr{D}\left(f\left(x_{0}\right), \omega\right), \mathscr{D}\left(x_{0}, f(\omega)\right)\right\} .
\end{gathered}
$$

By using (46) and (47) we have

$$
\begin{aligned}
\mathscr{D}\left(x_{0}, \omega\right) & \leq C k^{0} \delta\left(\mathscr{D}, f, x_{0}\right)=C \delta\left(\mathscr{D}, f, x_{0}\right), \\
\mathscr{D}\left(x_{0}, f\left(x_{0}\right)\right) & \leq k^{0} \delta\left(\mathscr{D}, f, x_{0}\right)=\delta\left(\mathscr{D}, f, x_{0}\right), \\
\mathscr{D}\left(f\left(x_{0}\right), \omega\right) & \leq C k \delta\left(\mathscr{D}, f, x_{0}\right)<C \delta\left(\mathscr{D}, f, x_{0}\right) .
\end{aligned}
$$

Hence, we derive

$$
\begin{aligned}
& \mathscr{D}\left(f\left(x_{0}\right), f(\omega)\right) \leq \max \left\{k C \delta\left(\mathscr{D}, f, x_{0}\right),\right. \\
& \left.\quad k \delta\left(\mathscr{D}, f, x_{0}\right), k \mathscr{D}(\omega, f(\omega)), k \mathscr{D}\left(x_{0}, f(\omega)\right)\right\} .
\end{aligned}
$$

Again by using the fact that $f$ is generalized $k$-quasicontraction mapping and the technique used above, we observe that

$$
\begin{aligned}
\mathscr{D} & \left(f^{2}\left(x_{0}\right), f(\omega)\right) \leq \max \left\{k^{2} C \delta\left(\mathscr{D}, f, x_{0}\right),\right. \\
& \left.k^{2} \delta\left(\mathscr{D}, f, x_{0}\right), k \mathscr{D}(\omega, f(\omega)), k^{2} \mathscr{D}\left(x_{0}, f(\omega)\right)\right\} .
\end{aligned}
$$

By continuing in the same manner, we deduce

$$
\begin{aligned}
\mathscr{D} & \left(f^{n}\left(x_{0}\right), f(\omega)\right) \leq \max \left\{k^{n} C \delta\left(\mathscr{D}, f, x_{0}\right),\right. \\
& \left.k^{n} \delta\left(\mathscr{D}, f, x_{0}\right), k \mathscr{D}(\omega, f(\omega)), k^{n} \mathscr{D}\left(x_{0}, f(\omega)\right)\right\},
\end{aligned}
$$

for every $n \in \mathbb{N}$. Now, as $\mathscr{D}\left(x_{0}, f(\omega)\right)<\infty$ and $\delta\left(\mathscr{D}, f, x_{0}\right)<$ $\infty$, we have

$$
\limsup _{n \rightarrow \infty} \mathscr{D}\left(f^{n}\left(x_{0}\right), f(\omega)\right) \leq k \mathscr{D}(\omega, f(\omega)) .
$$

Regarding the condition $\left(\mathrm{D}_{2}\right)$ and the fact that $C<1$ and $k<1$, we get

$$
\begin{aligned}
\mathscr{D}(\omega, f(\omega)) & \leq C \limsup _{n \rightarrow \infty} \mathscr{D}\left(f^{n}\left(x_{0}\right), f(\omega)\right) \\
& \leq C k \mathscr{D}(\omega, f(\omega))<\mathscr{D}(\omega, f(\omega)) .
\end{aligned}
$$

Thus, $\mathscr{D}(\omega, f(\omega))=0$. By analogy, as in the above, we can conclude that

$$
\mathscr{D}(f(\omega), \omega)<\mathscr{D}(\omega, f(\omega))=0 .
$$

Hence, we have $\mathscr{D}(\omega, f(\omega))=\mathscr{D}(f(\omega), \omega)=0$. Therefore, we get $\omega=f(\omega)$; that is, $\omega$ is a fixed point of $f$.

Now, if $\omega^{\prime}$ is another fixed point of $f$ with $\mathscr{D}\left(\omega^{\prime}, \omega\right)<\infty$, $\mathscr{D}\left(\omega, \omega^{\prime}\right)<\infty$, and $\mathscr{D}\left(\omega^{\prime}, \omega^{\prime}\right)<\infty$, then as $f$ is generalized $k$-quasi contraction mapping we have

$$
\begin{aligned}
\mathscr{D}\left(\omega^{\prime}, \omega\right) & \mathscr{D}\left(f\left(\omega^{\prime}\right), f(\omega)\right) \leq k \\
\cdot & \max \left\{\mathscr{D}\left(\omega^{\prime}, \omega\right), \mathscr{D}\left(\omega^{\prime}, \omega^{\prime}\right), \mathscr{D}(\omega, \omega), \mathscr{D}\left(\omega, \omega^{\prime}\right)\right\}
\end{aligned}
$$


as $\mathscr{D}(\omega, f(\omega))=\mathscr{D}(\omega, \omega)<\infty$ and $\mathscr{D}\left(\omega^{\prime}, \omega^{\prime}\right)<\infty$. So, by Proposition 30, we have

$$
\mathscr{D}(\omega, \omega)=\mathscr{D}\left(\omega^{\prime}, \omega^{\prime}\right)=0,
$$

which implies that $\mathscr{D}\left(\omega^{\prime}, \omega\right) \leq k \max \left\{\mathscr{D}\left(\omega^{\prime}, \omega\right), \mathscr{D}\left(\omega, \omega^{\prime}\right)\right\}$. In a similar way, we derive that

$$
\mathscr{D}\left(\omega, \omega^{\prime}\right) \leq k \max \left\{\mathscr{D}\left(\omega^{\prime}, \omega\right), \mathscr{D}\left(\omega, \omega^{\prime}\right)\right\},
$$

so that

$$
\begin{aligned}
\max & \left\{\mathscr{D}\left(\omega^{\prime}, \omega\right), \mathscr{D}\left(\omega, \omega^{\prime}\right)\right\} \\
\leq k \max & \left\{\mathscr{D}\left(\omega^{\prime}, \omega\right), \mathscr{D}\left(\omega, \omega^{\prime}\right)\right\} .
\end{aligned}
$$

Since $\mathscr{D}\left(\omega, \omega^{\prime}\right)<\infty, \mathscr{D}\left(\omega^{\prime}, \omega\right)<\infty$, and $k \in(0,1)$, we deduce that $\mathscr{D}\left(\omega^{\prime}, \omega\right)=\mathscr{D}\left(\omega, \omega^{\prime}\right)=0$ which yields $\omega=$ $\omega^{\prime}$.

\section{Fixed Point Theorems in Quasi-JS-Metric Space with Partial Order}

Definition 32. Let $(X, \mathscr{D})$ be a quasi-JS-metric space with partial order $\preceq$ and let $f: X \rightarrow X$ be a mapping. We say that $f$ is weakly continuous if the following condition holds: if $\left\{x_{n}\right\} \subseteq X$ is $\mathscr{D}$-convergent to $x \in X$, then there exists a subsequence $\left\{x_{n_{k}}\right\}$ of $\left\{x_{n}\right\}$ such that $\left\{f\left(x_{n_{k}}\right)\right\}$ is $\mathscr{D}$-convergent to $f(x)$ as $k \rightarrow \infty$.

Definition 33. Let $X$ be a nonempty set with partial order $\preceq$. A mapping $f: X \rightarrow X$ is said to be nondecreasing if

$$
x \preceq y \Longrightarrow f(x) \preceq f(y) \text { where } x, y \in X .
$$

Definition 34. The pair $(X, \preceq)$ is said to be $\mathscr{D}$-left regular (resp., $\mathscr{D}$-right regular) if the following condition holds: for every sequence $\left\{x_{n}\right\} \subseteq X$ satisfies $x_{n+1} \preceq x_{n}\left(x_{n} \preceq x_{n+1}\right)$ for each $n \in \mathbb{N}$, with $\left\{x_{n}\right\}$ being $\mathscr{D}$-convergent to $x \in X$; then there exists a subsequence $\left\{x_{n_{k}}\right\}$ of $\left\{x_{n}\right\}$ such that $x \preceq x_{n_{k}}$ $\left(x_{n_{k}} \preceq x\right)$ for every $k \in \mathbb{N}$.

The pair $(X, \preceq)$ is said to be $\mathscr{D}$-regular if and only if it is left and right $\mathscr{D}$-regular.

Definition 35. A function $f: X \rightarrow X$ is said to be weakly $k$-contraction if $x \preceq y$ or $y \preceq x$ implies

$$
\mathscr{D}(f(x), f(y)) \preceq k \mathscr{D}(x, y) .
$$

That is, whenever $x, y \in X$ are comparable condition (61) is satisfied.

Theorem 36. Let $(X, \mathscr{D})$ be a quasi-JS-metric space with partial order $\preceq$ and let $f: X \rightarrow X$ be a function. Assume that the following conditions hold:

(i) $(X, \mathscr{D})$ is $\mathscr{D}$-complete;

(ii) $f$ is weakly continuous;

(iii) $f$ is weakly $k$-contraction for some $k \in(0,1)$; (iv) $f$ is nondecreasing;

(v) there exists $x_{0} \in X$ such that $\delta\left(\mathscr{D}, f, x_{0}\right)<\infty$ and $x_{0} \preceq f\left(x_{0}\right)$.

Then $f$ has a fixed point $\omega \in X$ and $\left\{f^{n}\left(x_{0}\right)\right\}$ is $\mathscr{D}$ convergent to $\omega$. Moreover, if $\mathscr{D}(\omega, \omega)<\infty$, then $\mathscr{D}(\omega, \omega)=0$.

Proof. Since $f$ is nondecreasing and $x_{0} \preceq f\left(x_{0}\right)$, then for every $n \in \mathbb{N}_{0}$ we have

$$
f^{n}\left(x_{0}\right) \preceq f^{n+1}\left(x_{0}\right)
$$

and by the transitivity of $\preceq$, for every $p, q \in \mathbb{N}_{0}$, we have

$$
p \leq q \Longrightarrow f^{p}\left(x_{0}\right) \preceq f^{q}\left(x_{0}\right) \text {. }
$$

Therefore, for each $n \in \mathbb{N}, i, j \in \mathbb{N}_{0}, f^{n+i}\left(x_{0}\right)$ and $f^{n+j}\left(x_{0}\right)$ are always comparable. As $f$ is weak $k$-contraction for each $n \in \mathbb{N}, i, j \in \mathbb{N}_{0}$, we have

$$
\begin{aligned}
& \mathscr{D}\left(f^{n+i}\left(x_{0}\right), f^{n+j}\left(x_{0}\right)\right) \\
& \quad \leq k \mathscr{D}\left(f^{n-1+i}\left(x_{0}\right), f^{n-1+j}\left(x_{0}\right)\right) .
\end{aligned}
$$

Hence

$$
\delta\left(\mathscr{D}, f, f^{n}\left(x_{0}\right)\right) \leq k \delta\left(\mathscr{D}, f, f^{n-1}\left(x_{0}\right)\right) .
$$

Thus, for every $n \in \mathbb{N}_{0}$ we have

$$
\delta\left(\mathscr{D}, f, f^{n}\left(x_{0}\right)\right) \leq k^{n} \delta\left(\mathscr{D}, f, x_{0}\right) .
$$

Using the above inequality we have for every $m, n \in \mathbb{N}_{0}$

$$
\begin{aligned}
& \mathscr{D}\left(f^{n}\left(x_{0}\right), f^{n+m}\left(x_{0}\right)\right) \leq \delta\left(\mathscr{D}, f, f^{n}\left(x_{0}\right)\right) \\
& \quad \leq k^{n} \delta\left(\mathscr{D}, f, x_{0}\right), \\
& \mathscr{D}\left(f^{n+m}\left(x_{0}\right), f^{n}\left(x_{0}\right)\right) \leq \delta\left(\mathscr{D}, f, f^{n}\left(x_{0}\right)\right) \\
& \quad \leq k^{n} \delta\left(\mathscr{D}, f, x_{0}\right) .
\end{aligned}
$$

Since $\delta\left(\mathscr{D}, f, x_{0}\right)<\infty$ and $k \in(0,1)$ we have

$$
\begin{aligned}
\lim _{m, n \rightarrow \infty} \mathscr{D}\left(f^{n}\left(x_{0}\right), f^{n+m}\left(x_{0}\right)\right) \\
=\lim _{m, n \rightarrow \infty} \mathscr{D}\left(f^{n+m}\left(x_{0}\right), f^{n}\left(x_{0}\right)\right)=0,
\end{aligned}
$$

which means that $\left\{f^{n}\left(x_{0}\right)\right\}$ is right and left $\mathscr{D}$-Cauchy and hence $\mathscr{D}$-Cauchy sequence. By the completeness of $(X, \mathscr{D})$ there exists $\omega \in X$ such that $\left\{f^{n}\left(x_{0}\right)\right\}$ is $\mathscr{D}$-convergent to $\omega$. Since $f$ is weakly continuous, there exists a subsequence $\left\{f^{n_{k}}\left(x_{0}\right)\right\}$ of $\left\{f^{n}\left(x_{0}\right)\right\}$ such that $\left\{f^{n_{k}+1}\left(x_{0}\right)\right\}$ is $\mathscr{D}$-convergent to $f(\omega)$ as $k \rightarrow \infty$. By the uniqueness of the limit in a quasi$J S$-metric space we have $\omega=f(\omega)$ and $\omega$ is a fixed point of $f$.

Now, if $\mathscr{D}(\omega, \omega)<\infty$ then as $\omega \preceq \omega$ and $f$ is weak $k$ contraction we have

$$
\mathscr{D}(\omega, \omega)=\mathscr{D}(f(\omega), f(\omega)) \leq k \mathscr{D}(\omega, \omega),
$$

which is possible only if $\mathscr{D}(\omega, \omega)=0$. 
The weak continuity condition of $f$ in the previous theorem can be replaced by the regularity of the pair $(X, \preceq)$ as in the following result.

Theorem 37. Let $(X, \mathscr{D})$ be a quasi-JS-metric space with partial order $\preceq$ and let $f: X \rightarrow X$ be a function. Assume that the following conditions hold:

(i) $(X, \mathscr{D})$ is $\mathscr{D}$-complete;

(ii) $(X, \preceq)$ is regular;

(iii) $f$ is weakly $k$-contraction for some $k \in(0,1)$;

(iv) $f$ is nondecreasing;

(v) there exists $x_{0} \in X$ such that $\delta\left(\mathscr{D}, f, x_{0}\right)<\infty$ and $x_{0} \preceq f\left(x_{0}\right)$.

Then $f$ has a fixed point $\omega \in X$ and $\left\{f^{n}\left(x_{0}\right)\right\}$ is $\mathscr{D}$ convergent to $\omega$. Moreover, if $\mathscr{D}(\omega, \omega)<\infty$, then $\mathscr{D}(\omega, \omega)=0$.

Proof. Following the steps of the previous proof we can prove that $\left\{f^{n}\left(x_{0}\right)\right\}$ is $\mathscr{D}$-convergent to $\omega \in X$. Moreover, we have

$$
f^{n}\left(x_{0}\right) \preceq f^{n+1}\left(x_{0}\right)
$$

for every $n \in \mathbb{N}_{0}$. Since $(X, \preceq)$ is regular it is right regular and so there exists a subsequence $\left\{f^{n_{k}}\left(x_{0}\right)\right\}$ of $\left\{f^{n}\left(x_{0}\right)\right\}$ such that $f^{n_{k}}\left(x_{0}\right) \preceq \omega$ for each $k \in \mathbb{N}$. As $f$ is weakly $k$-contraction we have

$$
\mathscr{D}\left(f^{n_{k}+1}\left(x_{0}\right), f(\omega)\right) \leq \mathscr{D}\left(f^{n_{k}}\left(x_{0}\right), \omega\right)
$$

Using the inequality above, we get

$$
\lim _{k \rightarrow \infty} \mathscr{D}\left(f^{n_{k}+1}\left(x_{0}\right), f(\omega)\right)=0 .
$$

Similarly, we can prove that

$$
\lim _{k \rightarrow \infty} \mathscr{D}\left(f(\omega), f^{n_{k}+1}\left(x_{0}\right)\right)=0,
$$

which implies that $f(\omega)$ is a $\mathscr{D}$-limit for the sequence $\left\{f^{n_{k}+1}\left(x_{0}\right)\right\}$. By Proposition $4, f(\omega)=\omega$; and $\omega$ is a fixed point of $f$. As in the previous proof

$$
\mathscr{D}(\omega, \omega)<\infty \Longrightarrow \mathscr{D}(\omega, \omega)=0 \text {. }
$$

Example 38. Let $(X, \mathscr{D})$ be the complete quasi-JS-metric space introduced in Example 7. Define the function $f: X \rightarrow$ $X$ by

$$
f(x)= \begin{cases}\infty & \text { if } x=\infty \\ \frac{x}{2} & \text { otherwise }\end{cases}
$$

Then clearly $f$ is a $k$-contraction mapping with $k=1 / 2$. Let $x_{0}=0$ then $\delta\left(\mathscr{D}, f, x_{0}\right)<\infty$. So by Theorem $26 f$ has a fixed point.

\section{Competing Interests}

The authors declare that they have no competing interests.

\section{Authors' Contributions}

All authors contributed equally and significantly in writing this paper. All authors read and approved the final paper.

\section{References}

[1] M. Jleli and B. Samet, "A generalized metric space and related fixed point theorems," Fixed Point Theory and Applications, vol. 2015, article 61, 2015.

[2] H. P. Künzi, H. Pajoohesh, and M. P. Schellekens, "Partial metric space," Applied General Topology, vol. 6, no. 2, pp. 229-240, 2005.

[3] M. H. Shah and N. Hussain, "Nonlinear contractions in partially ordered quasi b-metric spaces," Communications of the Korean Mathematical Society, vol. 27, no. 1, pp. 117-128, 2012.

[4] F. M. Zeyada, G. H. Hassan, and M. A. Ahmad, "A generalization of fixed point theorem due to Hitzler and Seda in dislocated, quasi metric space," Arabian Journal for Science and Engineering, vol. 31, pp. 111-114, 2005.

[5] W. M. Kozlowski, Modular Function Space, vol. 122 of Monographs and Textbooks in Pure and Applied Mathematics, Marcel Dekker, New York, NY, USA, 1988. 


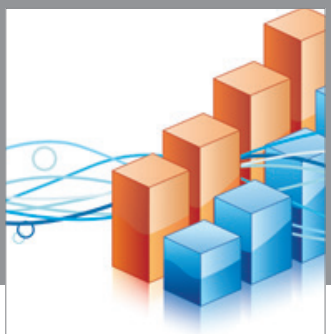

Advances in

Operations Research

vatem alat4

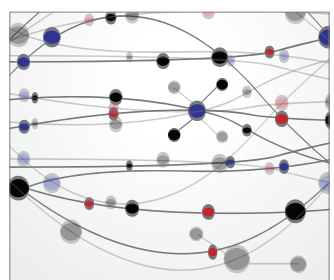

\section{The Scientific} World Journal
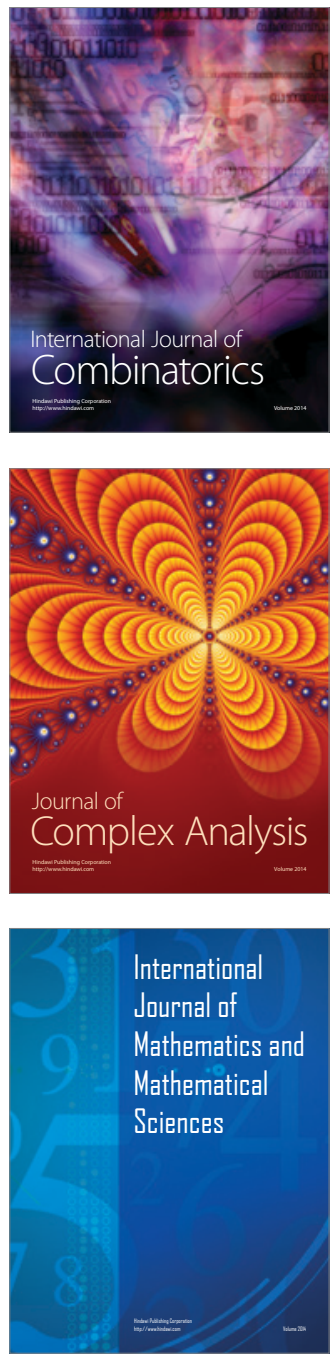
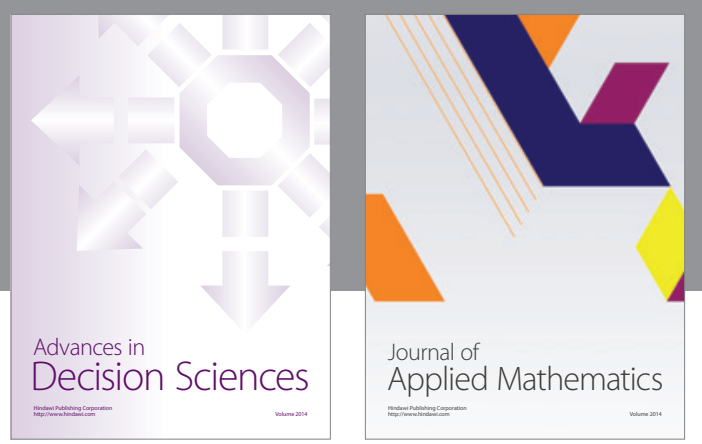

Algebra

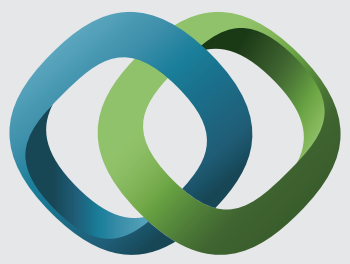

\section{Hindawi}

Submit your manuscripts at

http://www.hindawi.com
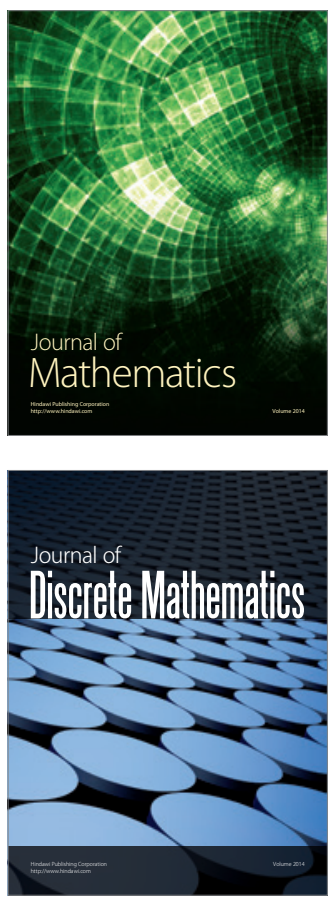

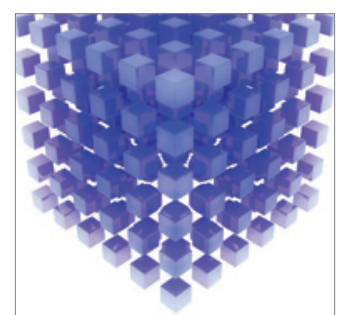

Mathematical Problems in Engineering
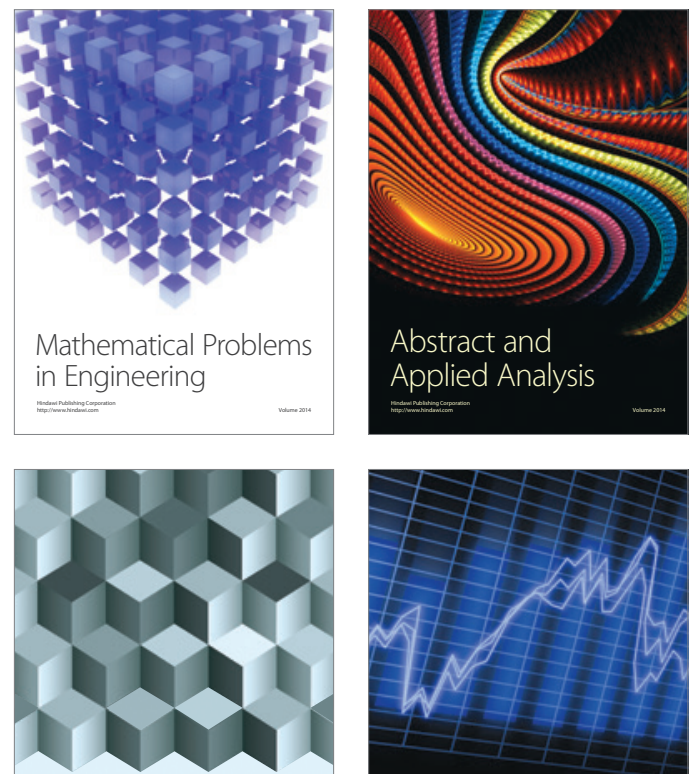

Journal of

Function Spaces

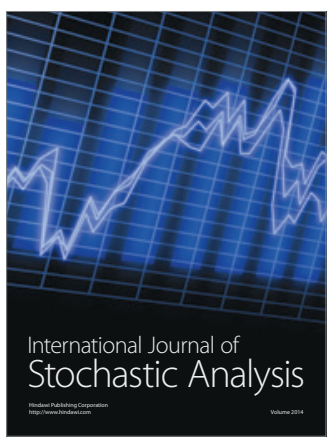

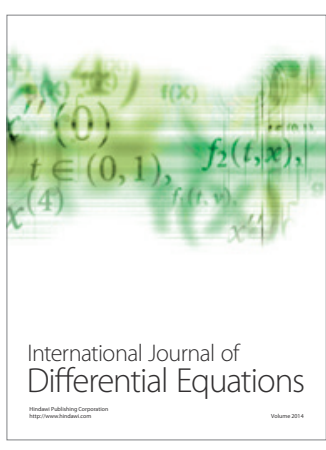
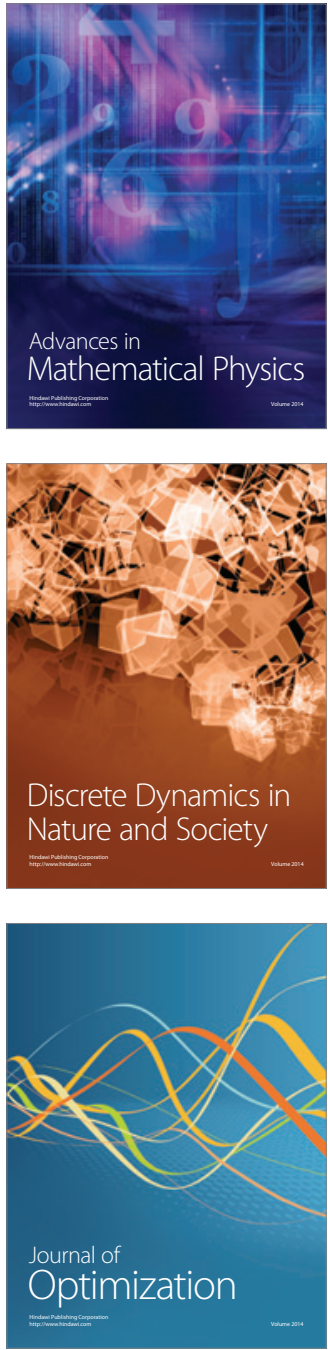